\title{
The Boltzmann-Grad Limit for a One-Dimensional Boltzmann Equation in a Stationary State
}

\author{
S. Caprino ${ }^{1}$, M. Pulvirenti ${ }^{2}$ \\ ${ }^{1}$ Dipartimento di Matematica, Università di Roma Tor Vergata, Rome, Italy \\ 2 Dipartimento di Matematica, Università di Roma La Sapienza, Rome, Italy
}

Received: 31 October 1994/in revised form: 28 April 1995

\begin{abstract}
In this paper we consider a one-dimensional model of interacting particles in a bounded interval with (possibly not homogeneous) diffusive boundary conditions. We prove that, when the number of particles $N$ goes to infinity and the interaction is suitably rescaled (the Boltzmann-Grad limit), the one-particle distribution function of the unique invariant measure for the particle system, converges to the unique solution of the Boltzmann equation of the model, provided that the mean free path is sufficiently large.
\end{abstract}

\section{Introduction}

One of the most important problems in nonequilibrium statistical mechanics is the analysis of stationary nonequilibrium states. For instance one can couple the system under consideration to thermal reservoirs which are maintained at different constant temperatures. After an initial transient time, the system is expected to approach a stationary state in which there is a steady flux of energy from the hot to the cold reservoir.

Such a state can be described at various levels: macroscopically by means of the hydrodynamical equations, mezoscopically, through the kinetic (Boltzmann) equations, microscopically according to the basic Liouville equation. The three levels of descriptions are related by various scaling limits some of which (see for instance [1] as regards the deduction of the stationary hydrodynamical solutions from the Boltzmann equation) have been successfully investigated. However very little is known about stationary nonequilibrium states in the microscopic description. Namely what we know about stationary solutions of the Liouville equation for realistic systems with a non-constant profile of temperature on the boundary is, at most, existence and uniqueness (see e.g. [2]), without any additional property which could allow to go further in analyzing the scaling limits. Even worse is our knowledge as regards the Boltzmann equation: we have only partial existence results (see [3-5]) for stationary nonequilibrium solutions. In this situation a rigorous analysis of the Boltzmann-Grad limit seems hopeless, that is a derivation of the stationary 
Boltzmann equation from the stationary solutions of the Liouville equation under a suitable scaling. Therefore a careful analysis of simplified models is useful, in which some of the features of more realistic systems are maintained and for which the problem can more easily be settled.

In this paper we want to study a one-dimensional model of interacting particles proposed by Ianiro and Lebowitz in [6] for the study of the stationary solutions of the corresponding Boltzmann equation. We prove that, under suitable assumptions and for large values of the mean free path, the stationary solutions of the Liouville (Master) equation, converge in the Boltzmann-Grad limit, to the (unique) solution of the Boltzmann equation.

The basic ingredient of our proof is a cluster expansion technique used by the same authors of the present paper for the time dependent problem (see [7]) on the whole line, which allows us the control of the interaction, combined with a mixing property of the free (Knudsen) flow. The full use of this last property makes necessary a lower bound for the allowed velocities which is, probably, only a technical restriction.

The paper is organized as follows. In Sect. 2 we introduce the model. In Sect. 3 we establish some preliminary results and the technical setup necessary for the proof of the main result. Here we introduce the $v$-functions, a sort of truncated correlation functions (see $[8,9]$ and references quoted therein), which play an important role in the convergence proof presented in Sect. 4. Two technical Appendices conclude the paper.

Some final comments are in order. After Lanford's result [10], it is well known that the Boltzmann equation can be rigorously derived, for short times, in terms of the classical particle dynamics (see also [11] for further comments and results). Even proving the validity of the Boltzmann equation for arbitrary times (but this seems much beyond our present knowledge) it is strongly doubtful whether such a validation could work uniformly in time, for general situations. As a consequence, even knowing the trend to a nonequilibrium stationary state for the Boltzmann dynamics (which is, incidentally, not known) we could not conclude anything about the approximation of the nonequilibrium stationary state in terms of the corresponding invariant state for the microscopic dynamics in the Boltzmann-Grad limit since we are not authorized to commute this limit with $t \rightarrow \infty$.

In this paper we approach the problem differently for the simplified model under consideration. We evaluate the distance between the invariant measure for the particle system and the stationary solution to the Boltzmann equation (which both are known to exist), in terms of the $v$-functions and find that the time invariance condition on a scale of time sufficiently large to take advantage of the mixing property of the free flow, and sufficiently small to control the interaction (for a sufficiently large mean free path), gives rise to a contraction property which allows us to achieve the proof.

To our knowledge this is the first result in this direction for a non-trivial model. Our assumptions allow us to work without knowing much about the microscopic invariant state and we believe that extensions including more realistic models are indeed very difficult. Our analysis is essentially perturbative, therefore it works for a large mean free path. One can ask whether the present result can be extended for arbitrary mean free paths. A preliminary problem to solve is then to prove (or disprove) the uniqueness of the stationary solution to the Boltzmann equation without any smallness assumption. This problem has not yet been solved even in our simplified model (see [6]). 


\section{The Model}

Let us consider a system of $N$ identical particles in the interval $[0,1]$. Denote by $Z_{N}=\left(X_{N}, V_{N}\right)=\left\{x_{1} v_{1}, \ldots, x_{N} v_{N}\right\}$ a state of the system, where $x_{i}$ and $v_{i}$ are the position and the velocity of the $i^{\text {th }}$ particle. $Z_{N} \in \Lambda_{N}=[0,1]^{N} \times \mathscr{V}^{N}$, the phase space of the system, where $\mathscr{V}=[-2,-1] \cup[1,2]$ is the set of all possible velocities. A collision between two particles is defined in the following way. Denoting by $v_{1}, v_{2}$ and by $v_{1}^{\prime}, v_{2}^{\prime}$ the ingoing and the outgoing velocities respectively, then

$$
\begin{gathered}
v_{1}^{\prime}=-v_{2}, \quad v_{2}^{\prime}=-v_{1} \quad \text { if } \operatorname{sgn}\left(v_{1}\right) \neq \operatorname{sgn}\left(v_{2}\right), \\
v_{1}^{\prime}=v_{1}, \quad v_{2}^{\prime}=v_{2} \quad \text { if } \operatorname{sgn}\left(v_{1}\right)=\operatorname{sgn}\left(v_{2}\right) .
\end{gathered}
$$

Notice that, due to the fact that the particles are identical, the above collision rule is equivalent to a specular reflection of each colliding particle. However we prefer a labelling for which the direction of the velocity does not change in the collision because this will simplify some technicalities later on. Moreover, according to (2.1), collisions between particles travelling in the same direction are not considered.

Notice also that the relative velocity is preserved during the collision:

$$
\left|v_{i}-v_{j}\right|=\left|v_{i}^{\prime}-v_{j}^{\prime}\right| \text {. }
$$

The dynamics of the system is stochastic. The particles move freely up to the first time in which two of them arrive at the same point. Then they collide (independently) with probability $\varepsilon$ and go ahead with probability $1-\varepsilon$. Moreover each particle is stochastically reflected by the boundary $\{0\} \cup\{1\}$ according to two given probability distributions, $\tilde{H}_{0}$ and $\tilde{H}_{1}$, for the outgoing velocities from 0 and 1 respectively. Namely, if a particle hits 0 (obviously with negative velocity) it is reflected with positive velocity $v$ distributed according to the distribution $\tilde{H}_{0}:[1,2] \rightarrow \mathbb{R}^{+}$with $\int_{1}^{2} d v \tilde{H}_{0}=1$. The negative velocity outgoing from a collision in 1 is distributed according to the distribution $\tilde{H}_{1}:[-2,-1] \rightarrow \mathbb{R}^{+}$with $\int_{-2}^{-1} d v \tilde{H}_{1}=1$.

The distributions $\tilde{H}_{0}$ and $\tilde{H}_{1}$ play the role of the Maxwellians of the walls and mimic two reservoirs at possibly different temperatures.

The stochastic process introduced above defines a semigroup $P_{N}^{t}$ in the following way. Let $v_{0}=v_{0}\left(Z_{N}\right)$ be a symmetric probability density. Then $v\left(Z_{N}, t\right)=P_{N}^{t} v_{0}\left(Z_{N}\right)$ is the time evolved measure defined by

$$
\int v\left(Z_{N}, t\right) \varphi\left(Z_{N}\right) d Z_{N}=\int v_{0}\left(Z_{N}\right) \mathbb{E} \varphi\left(T^{t} Z_{N}\right) d Z_{N},
$$

where $\varphi$ is any smooth test function, $T^{t} Z_{N}$ is the $N$-particle stochastic process starting almost surely from $Z_{N}$ and $\mathbb{E}$ denotes the expectation. It is easy to see that $P_{N}^{t}$ acts isometrically from $L_{1}\left(d Z_{N}\right)$ into itself. Formally $v$ satisfies

$$
\left(\partial_{t}+\sum_{i=1}^{N} v_{i} \partial_{i}\right) v\left(Z_{N}, t\right)=\frac{1}{2} \varepsilon \sum_{i \neq j} \delta\left(x_{i}-x_{j}\right) \chi(i, j)\left|v_{i}-v_{j}\right|\left\{v\left(Z_{N}^{\prime}(i, j), t\right)-v\left(Z_{N}, t\right)\right\},
$$

where $\partial_{i}$ is the derivative with respect to $x_{i}, \delta(\cdot)$ is the $\delta$-function centered at zero. $\chi(i, j)=1$ if $\operatorname{sgn}\left(v_{i}\right) \neq \operatorname{sgn}\left(v_{j}\right)$ and 0 otherwise,

$$
Z_{N}^{\prime}(i, j)=\left(x_{1} v_{1}, \ldots, x_{i} v_{i}^{\prime}, \ldots, x_{j} v_{j}^{\prime}, \ldots, x_{N} v_{N}\right)
$$


if

$$
Z_{N}=\left(x_{1} v_{1}, \ldots, x_{i} v_{i}, \ldots, x_{j} v_{j}, \ldots, x_{N} v_{N}\right) .
$$

Equation (2.4) must be complemented by the initial condition

$$
v\left(Z_{N}, 0\right)=v_{0}\left(Z_{N}\right),
$$

and the boundary conditions which we are going to make explicit. To this purpose it is convenient to introduce

$$
\begin{aligned}
& \mathscr{F}_{0}^{i}=\left\{Z_{N} \in \Lambda_{N} \mid x_{i}=0\right\}, \\
& \mathscr{F}_{1}^{i}=\left\{Z_{N} \in \Lambda_{N} \mid x_{i}=1\right\} .
\end{aligned}
$$

Then for $\left(X_{N}, V_{N}\right) \in \mathscr{F}_{0}^{i}$ with $v_{i}>0, V_{N}=\left\{v_{1}, \ldots, v_{N}\right\}$ we have

$$
v\left(X_{N}, V_{N}, t\right)=-H_{0}\left(v_{i}\right) \int_{w_{l}<0} w_{i} v\left(X_{N}, v_{1} \cdots w_{i} \cdots v_{N}, t\right) d w_{i} .
$$

For $\left(X_{N}, V_{N}\right) \in \mathscr{F}_{1}^{i}$ with $v_{i}<0$ we have

$$
v\left(X_{N} V_{N}, t\right)=H_{1}\left(v_{i}\right) \int_{w_{i}>0} w_{i} v\left(X_{N}, v_{1} \cdots w_{i} \cdots v_{N}, t\right) d w_{i} .
$$

The above boundary conditions are written in terms of $H_{0}$ and $H_{1}$. They are related to the Maxwellians $\tilde{H}_{0}$ and $\tilde{H}_{1}$ by a constant, in such a way that

$$
\int H_{i}(v) v d v=1, \quad i=1,2,
$$

that is $H_{0,1}=\tilde{H}_{0,1} / \int_{v \gtrless 0} v \tilde{H}_{0,1} d v$. In this way the conservation of the probability is guaranteed.

It is a consequence of general arguments from the theory of Markov processes (see [2]) that $P_{N}^{t}$ has a unique invariant measure, denoted by $\mu^{N}$, which is also the (formal) solution of the equation:

$$
\sum_{i=1}^{N} v_{i} \partial_{i} \mu^{N}\left(Z_{N}\right)=\frac{1}{2} \varepsilon \sum_{i \neq j} \delta\left(x_{i}-x_{j}\right) \chi(i, j)\left|v_{i}-v_{j}\right|\left\{\mu^{N}\left(Z_{N}^{\prime}(i, j)\right)-\mu\left(Z_{N}\right)\right\}
$$

In this paper we are interested in the behavior of the stationary solution $\mu^{N}$ in the limit $N \rightarrow \infty, \varepsilon \rightarrow 0$ in such a way that the combination $\varepsilon N=\lambda$ is constant. We call this limit the Boltzmann-Grad limit. The expected result is that the measure $\mu^{N}$ is going to factorize in this limit (propagation of chaos) and that the one-particle distribution function approximates a solution of the stationary Boltzmann equation relative to the model under consideration. This is given by

$$
v \partial_{x} g(x, v)=\lambda \int d v_{1}\left|v-v_{1}\right|\left\{g\left(x, v^{\prime}\right) g\left(x, v_{1}^{\prime}\right)-g(x, v) g\left(x, v_{1}\right)\right\},
$$

where the integral is on the set $\operatorname{sgn}\left(v_{1}\right) \neq \operatorname{sgn}(v)$, with the boundary conditions

$$
g(0, v)=-H_{0}(v) \int_{w<0} w g(0, w) d w, \quad v>0,
$$

and

$$
g(1, v)=H_{1}(v) \int_{w>0} w g(1, w) d w, \quad v<0 .
$$

We also fix the total mass equal to one:

$$
\int g(x, v) d x d v=1 .
$$


The solutions to the above Boltzmann equation have been investigated by Ianiro and Lebowitz in [6] by rather explicit computations. In particular they obtained existence of bounded solutions and uniqueness for $\lambda$ small.

To give a formal idea of how to get the Boltzmann-Grad limit for this system, it is convenient to introduce the $j$-particle distribution functions for the measure $\mu^{N}$ and introduce a hierarchy of equations (the BBKGY hierarchy of the model) as for the time dependent case (see [7]). Then assuming the propagation of chaos (indeed this property must be proven), one can check that the formal limit holds. This heuristic derivation is standard and absolutely identical to the time dependent case so that we omit it here.

\section{Definitions and Preliminaries}

For a fixed $N$, we denote by $I_{k}=\{1,2, \ldots, k\}, k \leqq N$, the set of the first $k$ integers while $I, J$, etc. will stand for any subset of indices of $I_{N}$. We will use also the notation $Z_{I}=\left\{x_{i}, v_{i}\right\}_{i \in I}$ and $|I|$ for the cardinality of $I$.

We denote by $K^{t} Z_{I}$ the Knudsen process, that is free dynamics of the group of particles with indices in $I$, starting almost surely from $Z_{I}$, with the diffusive boundary conditions described in the previous sections. In other words $K^{t} Z_{I}$ is the same as $T^{t} Z_{I}$ for $\varepsilon=0$.

Associated to $K^{t}$ we also introduce $\bar{P}_{I}^{t}$ the (Knudsen) semigroup defined by

$$
\left\langle\bar{P}_{I}^{t} f, \varphi\right\rangle=\int f\left(Z_{I}\right) \mathbb{E}\left(\varphi\left(K^{t} Z_{I}\right)\right) d Z_{I}
$$

for $f \in L_{1}$ and $\varphi \in L_{\infty}$. It is easy to see that $\bar{P}_{I}^{t}$ acts isometrically from $L_{1}\left(d Z_{I}\right)$ into itself.

We now establish an important asymptotic property of the free flow $K^{t}$ which will be crucial in what follows.

Proposition 3.1. For all $\eta>0$ there exists $t_{0}>0$, such that for all $t>t_{0}$ and all $u \in L_{1}\left(d Z_{I}\right)$ such that

$$
\int u\left(Z_{I}\right) d z_{i}=0, \quad i \in I,
$$

then

$$
\left\|\bar{P}_{I}^{t} u\right\|_{1} \leqq \eta^{j}\|u\|_{1},
$$

where $j=|I|$ and \|\|$_{1}$ denotes the $L_{1}$ norm.

Proposition 3.1 follows by a careful analysis of the Knudsen flow carried out by Arkeryd et al. in [12]. Notice that, in proving (3.3) we actually need the condition $|v| \geqq c>0$. For further comments, see Appendix A where the proof of Proposition 3.1 is sketched.

We now discuss the second preliminary result which we need later on. Let $g_{j}$ denote the $j$-fold product of solutions of the stationary Boltzmann equation (2.8), i.e.

$$
g_{j}\left(x_{1} v_{1} \cdots x_{j} v_{j}\right)=\prod_{i=1}^{j} g\left(x_{i}, v_{i}\right)
$$

Denote by $g_{j}^{N}(t)$ the $j$-particle distribution function of the symmetric measure $P_{N}^{t} g_{N}$, that is

$$
g_{j}^{N}\left(x_{1} v_{1} \cdots x_{j} v_{j} ; t\right)=\int d x_{j+1} d v_{j+1} \cdots d x_{N} d v_{N} P_{N}^{t} g_{N}\left(x_{1} v_{1} \cdots x_{N} v_{N}\right) .
$$


Setting

$$
r_{j}^{N}(t)=g_{j}^{N}(t)-g_{j}
$$

we have:

Proposition 3.2. There exist positive constants $A, B$ and $D$ such that if

$$
\lambda\|g\|_{\infty} \tau \leqq A e^{-B \tau},
$$

then

$$
\sup _{0 \leqq t \leqq \tau}\left\|r_{j}^{N}(t)\right\|_{1} \leqq \frac{e^{D j}}{N} .
$$

The proof is based on a Lanford type argument (see $[10,11]$ ) and will be proven in Appendix B.

Notice that the stationary solution $g$ found in [6] obviously depends on the parameter $\lambda$. However from [6] we also argue that $\|g\|_{\infty}$ is uniformly bounded for $\lambda$ in a neighbourhood of the origin so that (3.7) is certainly satisfied for $\lambda$ small.

We now introduce the important notion of cluster which will play an important role in the sequel. With this scope, as in [7], we find it convenient to give a partially explicit representation of the stochastic process $T^{t}$ in a suitable sample space. Define

$$
\Omega=\{0,1\}^{\frac{r N(N-1)}{2}},
$$

where $r$ is a positive integer. Then $\omega \in \Omega, \omega=\omega(i, j ; \alpha)=\omega(j, i ; \alpha), i \neq j$, where $\alpha=1 \ldots r$, is a function defined on the set of all pairs of particles and all integers less than or equal to $r$, taking values 0 and 1 . Define the stochastic process

$$
T_{\omega}^{t}: \Lambda_{N} \rightarrow \Lambda_{N} ; \quad t \leqq \frac{r-1}{4}
$$

in the following way. $T_{\omega}^{t} Z_{N}$ is the free motion with diffusive boundary conditions (that is the $N$-particle Knudsen flow) unless at time $t$ two particles, say $i$ and $j$, are at the same point with opposite velocities. Suppose that in the time interval $[0, t)$ they have met, with opposite velocities, $\alpha-1$ times. Then if $\omega(i, j ; \alpha)=1$, the two particles collide according to the law (2.1), otherwise they keep their free motion, up to the next collision. In other words $T_{\omega}^{t}$ is deterministic as regards the collisions among the particles and stochastic as regards the collisions with the boundary. Notice that, due to the boundedness of the velocities, each pair of particles can meet at most $r$ times up to the time $t \leqq \frac{r-1}{4}$.

$T_{\omega}^{t}$ is almost everywhere defined with respect to the Lebesgue measure on $\Lambda_{N}$. Indeed it is not defined on the sets of all $Z_{N}$ 's which deliver triple collisions and on the set of configurations for which, for some $i, j \in N$, it is $x_{i}=x_{j}, \operatorname{sgn}\left(v_{i}\right) \neq \operatorname{sgn}\left(v_{j}\right)$ and $\omega(i, j ; 1)=1$, being, in this last case, not possible to distinguish between preand post-collisional configurations. However these sets have measure zero so that we disregard them.

Notice also that $T_{\omega}^{t}$ is not a Markov process because to know whether a collision between two given particles takes place, we must know how many times the two particles have met in the past.

We define the probability of a single event $\omega \in \Omega$ by

$$
p(\omega)=\prod_{i, j} \prod_{\alpha=1}^{r} \varepsilon^{\omega(i, j ; \alpha)}(1-\varepsilon)^{1-\omega(i, j ; \alpha)},
$$


where $\prod_{i, j}$ denotes the product on all pairs. $\{\Omega, p\}$ is a probability space and we can write:

$$
\mathbb{E}\left(\varphi\left(T^{t} Z_{N}\right)\right)=\mathbb{E}_{\Omega} \mathbb{E}_{B}\left(\varphi\left(T_{\omega}^{t} Z_{N}\right)\right),
$$

where $\varphi$ is a test function, $\mathbb{E}_{B}$ denotes the expectation with respect to the distributions of the outgoing velocities after the collisions with the boundary and $\mathbb{E}_{\Omega}$ is the expectation with respect to $\{\Omega, p\}$.

Given $\omega \in \Omega$, we denote by $\operatorname{ch}(i)$ ("chain" starting from $i$ ) any set $I \subset I_{N} / i$ of indices such that

$$
\omega\left(i, i_{1} ; \alpha_{1}\right) \omega\left(i_{1}, i_{2} ; \alpha_{2}\right) \cdots \omega\left(i_{k-1}, i_{k} ; \alpha_{k}\right)=1
$$

for some ordering $i_{1}, \ldots, i_{k}$ of the set $I$ and some sequence $\alpha_{1} \cdots \alpha_{k}, \alpha_{i}=1 \cdots r$. The union of all chains starting from the particle $i$ will be called "cluster of $i$ " and denoted by

$$
\operatorname{cl}(i)=\bigcup \operatorname{ch}(i)
$$

Notice that the index $i$ is not included in $\operatorname{cl}(i)$. Moreover we denote by

$$
\operatorname{cl}(I)=\bigcup_{i \in I} \operatorname{cl}(i) / I
$$

the cluster of the set $I$. Notice that $\operatorname{cl}(I)$ is the set of all particles which can influence the dynamics of the set $I$ (excluded the set $I$ itself).

Finally we define

$$
n(I)=\sum_{i, j \in I} \sup _{1 \leqq \alpha \leqq r} \omega(i, j ; \alpha),
$$

that is the number of the active (interacting) pairs among the particles in $I$.

We conclude the notational part of this section by introducing the so-called $v$-functions.

We define (for $|I|=j$ ):

$$
f^{N}\left(Z_{I}\right)=\int \mu^{N}\left(Z_{I}, Z_{I_{N} / I}\right) d Z_{I_{N} / I}
$$

the $j$-particle distribution function of the symmetric measure $\mu^{N}$, the unique invariant measure of the process $T^{t}$. We shall also use the equivalent notation $f^{N}\left(Z_{I}\right) \equiv f_{j}^{N}\left(Z_{I}\right) \equiv f_{I}^{N}\left(Z_{I}\right)$. Moreover, let

$$
g\left(Z_{I}\right)=\prod_{i \in I} g\left(z_{i}\right)
$$

be the product of stationary solutions to the Boltzmann equation (see (3.4)). We set

$$
v^{N}\left(Z_{I}\right)=\sum_{J \subseteq I}(-1)^{|J|} g\left(Z_{J}\right) f^{N}\left(Z_{I / J}\right),
$$

where $\sum_{J \subseteq I}$ means the sum over all subsets of $I$ (including $I$ ), assuming also that

$$
v^{N}(\emptyset)=g(\emptyset)=f^{N}(\emptyset)=1 .
$$

Notice that $\sum_{J \subseteq I}(-1)^{|J|}=0$ if $I$ is not the empty set and 1 otherwise. Thus we have

$$
f^{N}\left(Z_{I}\right)=\sum_{J \subseteq I} g\left(Z_{J}\right) v^{N}\left(Z_{I / J}\right) .
$$


If $f^{N}$ would factorize

$$
f^{N}\left(Z_{I}\right)=\prod_{i \in I} f\left(z_{i}\right)
$$

then:

$$
v^{N}\left(Z_{I}\right)=\prod_{i \in I}\left(f\left(z_{i}\right)-g\left(z_{i}\right)\right) .
$$

The $v$-functions defined in (3.18) give a measure of the deviation of $f_{j}^{N}$ from $g_{j}$. Indeed if $v_{j}^{N} \rightarrow 0$ as $N \rightarrow \infty$, for all $j \geqq 1$ (as we shall prove for $\lambda=\varepsilon N$ small enough), then also $f_{j}^{N} \rightarrow g_{j}$ for all $j=1,2, \ldots$, as follows by (3.20) and (3.19). As we shall see later on, it seems more convenient to estimate the $v$-functions in place of the differences $f_{j}^{N}-g_{j}$ (roughly speaking the product of the differences rather than the differences of the products). Indeed by the property

$$
\int v^{N}\left(Z_{I}\right) d z_{i}=0 \quad i \in I .
$$

(which follows by the definition) and by Proposition 3.1, we have that

$$
\left\|\bar{P}_{I}^{t} v^{N}\right\|_{1} \leqq \eta^{j}\left\|v^{N}\right\|_{1}, \quad|I|=j,
$$

while the same arguments of Proposition 3.1 give nothing better than

$$
\left\|\bar{P}_{I}^{t}\left(f_{j}^{N}-g_{j}\right)\right\|_{1} \leqq \eta j\left\|\left(f_{j}^{N}-g_{j}\right)\right\|_{1} .
$$

Therefore $\left\|\bar{P}_{I}^{t} v^{N}\right\|_{1}$ decays, for fixed $t$ and $j$ large, much better than $\left\|\bar{P}_{I}^{t}\left(f_{j}^{N}-g_{j}\right)\right\|_{1}$, and this will be essential in the proof of the main theorem in the next section.

\section{The Main Result}

In this section we establish the main result of this paper, namely the convergence in the Boltzmann-Grad limit of the $j$-particle distribution functions associated to the stationary measure $\mu^{N}$ to the $j$-fold products of the stationary solution of the corresponding Boltzmann equation.

Theorem 4.1. For $\lambda=\varepsilon N$ sufficiently small:

$$
\lim _{N \rightarrow \infty}\left\|v_{j}^{N}\right\|_{1}=0
$$

and, consequently:

$$
\lim _{N \rightarrow \infty}\left\|f_{j}^{N}-g_{j}\right\|_{1}=0 \text {. }
$$

Proof. We use the shorthand notation:

$$
\left(g * v^{N}\right)\left(Z_{I}\right)
$$

for the right-hand side of (3.20). Accordingly

$$
v^{N}\left(Z_{I}\right)=\left(g^{\perp} * f^{N}\right)\left(Z_{I}\right),
$$

where

$$
g^{\perp}\left(Z_{I}\right)=(-1)^{|I|} g\left(Z_{I}\right)
$$


By the time invariance condition we have

$$
\left\langle f_{I}^{N}, \varphi_{j}\right\rangle=\int d Z_{N} \mu^{N}\left(Z_{N}\right) \mathbb{E}\left(\varphi\left(T^{t} Z_{N}\right)\right)
$$

where $\varphi_{j}=\varphi_{j}\left(Z_{I}\right)$ is a smooth test function, $j=|I|$ and we use the notation $\varphi\left(Z_{K}\right)=\varphi_{j}\left(Z_{I}\right)$ for $I \subseteq K$.

We now expand the expression in the right-hand side of (4.6) in clusters:

$$
\left\langle f_{I}^{N}, \varphi_{j}\right\rangle=\sum_{\substack{S \subseteq I_{N} \\ S \cap I=\emptyset}} \int d Z_{I \cup S} f^{N}\left(Z_{I \cup S}\right) \mathbb{E}\left(\varphi\left(T^{t} Z_{I \cup S}\right) \chi(\operatorname{cl}(I)=S)\right) .
$$

Here $\chi($ something) denotes the characteristic function of the event something. The right-hand side of (4.7) follows from the fact that, if $\chi(\operatorname{cl}(I)=S)=1$ then

$$
\varphi\left(T^{t} Z_{N}\right)=\varphi\left(T^{t} Z_{I \cup S}\right)
$$

since, by definition of cluster, the dynamics of the particles with labels in $(I \cup S)^{c}$ cannot influence those with labels in $I$.

Defining $\bar{f}_{I}^{N}(t)$ by

$$
\begin{aligned}
& \left\langle\bar{f}_{I}^{N}, \varphi_{j}\right\rangle=\sum_{\substack{S \subseteq I_{N} \\
S \cap I=\emptyset}} \sum_{\substack{S_{1} \cdots S_{j}: \\
\bigcup_{i} S_{i}=S \\
S_{r} \cap S_{k}=\emptyset, r \neq k}} \int d Z_{I \cup S} f^{N}\left(Z_{I \cup S}\right) \\
& \mathbb{E}\left(\varphi\left(T^{t} Z_{I \cup S}\right) \prod_{i \in I} \chi\left(\operatorname{cl}(i)=S_{i}\right) \chi\left(n\left(\bar{S}_{i}\right)=\left|S_{i}\right|\right)\right),
\end{aligned}
$$

where $\bar{S}_{i}=\{i\} \cup S_{i}$ and $n$ is given by (3.15), we have

$$
f_{I}^{N}=\bar{f}_{I}^{N}(t)+R_{I}^{1}(t)
$$

with

$$
\left\|R_{I}^{1}(t)\right\|_{1} \leqq \frac{C_{1}^{j}}{N}
$$

with some $C_{1}>0$ for $\lambda r$ sufficiently small.

The decomposition (4.10) and estimate (4.11.1) is analogous to those given in [7] (see (5.3) and (5.24)) which we shall comment later on, after estimate (4.31) of the present paper. For the moment we proceed algebraically. We operate on $g^{N}(t)$ the same decomposition as for $f^{N}$ :

$$
g_{I}^{N}(t)=\bar{g}_{I}^{N}(t)+R_{I}^{2}(t),
$$

where $\bar{g}_{I}^{N}$ is given by the analogous expansion (4.9) (here $g^{N}$ replaces $f^{N}$ ) and where

$$
\left\langle g_{I}^{N}(t), \varphi_{j}\right\rangle=\int d Z_{N} g^{N}\left(Z_{N}\right) \mathbb{E}\left(\varphi\left(T^{t} Z_{N}\right)\right), \quad|I|=j .
$$

Then

$$
\begin{aligned}
v^{N}\left(Z_{I}\right)= & \left(g^{\perp} * \bar{f}^{N}(t)\right)\left(Z_{I}\right)+\left(g^{\perp} * R^{1}(t)\right)\left(Z_{I}\right) \\
= & \left(\bar{g}^{N}(t)^{\perp} * \bar{f}^{N}(t)\right)\left(Z_{I}\right)+\left(g^{\perp} * R^{1}(t)\right)\left(Z_{I}\right) \\
& +\left(\left(g-g^{N}(t)\right)^{\perp} * \bar{f}^{N}(t)\right)\left(Z_{I}\right)+\left(R^{2}(t) * \bar{f}^{N}(t)\right)\left(Z_{I}\right) .
\end{aligned}
$$


Notice that the expansion (4.9) is done in terms of disjoint clusters with a minimal number of interacting pairs and that the remainder $R^{1}$ is negligible. Again from [7] we can prove

$$
\left\|R_{I}^{2}(t)\right\|_{1} \leqq \frac{C_{1}^{j}}{N}
$$

Thanks to estimates (4.11) and to the identity $R_{\emptyset}^{i}(t)=0, i=1,2$, the second and the fourth terms in the right-hand side of (4.14) vanish in the limit $N \rightarrow \infty$ in the $L_{1}$ sense and the same does the third term by Proposition 3.2. Thus we concentrate ourselves on the first term. Define $\bar{v}^{N}(t)$ by the following expression:

$$
\begin{gathered}
\left\langle\bar{v}_{I}^{N}(t), \varphi_{j}\right\rangle=\sum_{\substack{S \subseteq I_{N} \\
S \cap I=\emptyset}} \sum_{\substack{S_{1} \cdots S_{j}: \\
\bigcup_{i} \cap S_{i}=S}} \sum_{J \subseteq I}(-1)^{|J|} \\
\int d Z_{I \cup S} \mathbb{E}\left(\varphi\left(T^{t} Z_{I \cup S}\right) \prod_{i \in I} \chi\left(\operatorname{cl}(i)=S_{i}\right) \chi\left(n\left(\bar{S}_{i}\right)=\left|S_{i}\right|\right)\right) \\
f^{N}\left(\frac{\left.Z_{I \cup S(I / J)}^{J}\right)}{J}\right) g\left(Z_{J \cup S(J)}\right),
\end{gathered}
$$

where

$$
S(K)=\bigcup_{i \in K} S_{i}
$$

As we shall see at the end of this section, the difference

$$
R_{I}^{3}(t) \equiv \bar{g}_{I}^{N}(t)^{\perp} * \bar{f}_{I}^{N}(t)-\bar{v}_{I}^{N}(t)
$$

is vanishing as $N \rightarrow \infty$, so that the essential part in the right-hand side of (4.14) is just $\bar{v}^{N}(t)$ which we are going to estimate. To this purpose we introduce the norm

$$
\left\|v^{N}\right\|_{\alpha}=\sup _{I} e^{-\alpha|I|}\left\|v_{I}^{N}\right\|_{1}
$$

with $\alpha$ to be determined later on.

In (4.15) we write

$$
f^{N}\left(Z_{\frac{I \cup S(I / J)}{J}}\right) g\left(Z_{J \cup S(J)}\right)=\sum_{M \subseteq \frac{I \cup S(I / J)}{J}} v^{N}\left(Z_{M}\right) g\left(Z_{\frac{I \cup S}{M}}\right)
$$

and interchange the sums:

$$
\sum_{J \subseteq I} \sum_{M \subseteq \frac{I \cup S(I / J)}{J}}(-1)^{|J|}=\sum_{M \subseteq I \cup S} \sum_{J \subseteq \frac{I}{M \cup I(M)}}(-1)^{|J|}=\sum_{\substack{M \subseteq I \cup S \\ M \cup I(M) \supseteq I}}
$$

where

$$
I(M)=\left\{i \in I \mid i \notin M, S_{i} \cap M \neq \emptyset\right\}
$$


We now make explicit, in the expansion (4.15), those clusters $S_{i}$ which are empty:

$$
\begin{gathered}
\left\langle\bar{v}_{I}^{N}(t), \varphi_{j}\right\rangle=\sum_{k=0}^{j} \sum_{\substack{K \subset I \\
|K|=k}} \sum_{s \geqq j-k} \sum_{\substack{s_{l} \cdots s_{j}: \\
\sum^{s_{l}=s}}} \sum_{S \subseteq I_{N} / I} \sum_{\substack{S_{1} \cdots S_{j}: \\
\bigcup_{i} S_{i}=S \\
S_{r} \cap S_{k}=\emptyset, r \neq k \\
\left|S_{l}\right|=s_{i}}} \sum_{m \geqq j} \sum_{\substack{M \subseteq I \cup S \\
M \cup M(I) \supseteq I \\
|M|=m}} \\
\int d Z_{I \cup S} \mathbb{E}\left(\varphi_{j}\left(T^{t} Z_{I \cup S}\right) \prod_{i \in I} \chi\left(\operatorname{cl}(i)=S_{i}\right) \chi\left(n\left(\bar{S}_{i}\right)=s_{i}\right)\right. \\
\left.\prod_{i \in K^{c}} \chi(|\operatorname{cl}(i)|>0) \prod_{i \in K} \chi(\operatorname{cl}(i)=\emptyset)\right) v^{N}\left(Z_{M}\right) g\left(Z_{\frac{I \cup S}{M}}\right) .
\end{gathered}
$$

Notice that, since $M \cup M(I) \supseteq I$ then $m=|M| \geqq j$ and $K \subseteq M$. Indeed if $i \in I$, either $i \in M$ or $S_{i} \cap M \neq \emptyset$. Moreover

$$
\left(\varphi_{j}\left(T^{t} Z_{I \cup S}\right)\right)=\left(\varphi_{j}\left(K^{t} Z_{K}, T^{t} Z_{\frac{I \cup S}{K}}\right)\right)
$$

and, by Proposition 3.1 we can choose an arbitrary small $\eta$, to be chosen later, and a sufficiently large $t$ (and hence $r$ ), such that

$$
\left\|\bar{P}_{K}^{t} v_{M}^{N}\right\|_{1} \leqq \eta^{k}\left\|v_{M}^{N}\right\|_{1}, \quad|K|=k
$$

Therefore, from (4.22) we have the following estimate:

$$
\begin{aligned}
\left|\left\langle\bar{v}_{I}^{N}(t), \varphi_{j}\right\rangle\right| \leqq & \left\|\varphi_{j}\right\|_{\infty} \sum_{k=0}^{j} \eta^{k}\left(\begin{array}{l}
j \\
k
\end{array}\right) \sum_{s \geqq j-k} \sum_{\substack{s_{i} \cdots s_{j}: \\
\sum_{s_{i}=s}}} \sum_{m=j}^{j+s}\left(\begin{array}{c}
N \\
s
\end{array}\right)\left(\begin{array}{c}
j+s \\
m
\end{array}\right) \\
& \times \frac{s !}{s_{1} ! \cdots s_{j} !}\left\|v_{m}^{N}\right\|_{1}\left(\frac{\lambda r}{N}\right)^{s} \prod_{i=1}^{j} s_{i} ! e^{3 s} .
\end{aligned}
$$

Indeed the sums over the subsets give rise to the above combinatorial coefficients. To control the expectation

$$
\mathbb{E}\left(\prod_{i=1}^{j} \chi\left(\operatorname{cl}(i)=S_{i}\right) \chi\left(n\left(\bar{S}_{i}\right)=s_{i}\right)\right)
$$

we use the same argument as in [7]. The probability of having $\omega(i, j ; \alpha)=1$ for some $\alpha=1 \ldots r$ is bounded by $r \varepsilon$. So that (4.25) is bounded by $(r \lambda / N)^{s}$ times the number of ways we have to construct $j$ clusters with $s_{1} \cdots s_{j}$ given particles. Such a number has been estimated in [7] by $\prod_{i=1}^{j} s_{i} ! e^{3 s}$ (see (5.12) and (5.13) of [7]). From (4.25):

$$
\left\|\bar{v}_{I}^{N}(t)\right\|_{1} \leqq\left\|v^{N}\right\|_{\alpha} \sum_{k=0}^{j} \eta^{k}\left(\begin{array}{l}
j \\
k
\end{array}\right) e^{\alpha j} e^{j} 2^{j} \sum_{s \geqq j-k} s e^{\alpha s} e^{4 s} 2^{s}(\lambda r)^{s} .
$$


The above estimate follows by the elementary inequalities:

$$
\begin{gathered}
\left(\begin{array}{c}
N \\
s
\end{array}\right) \frac{s !}{N^{s}} \leqq 1, \quad \sum_{\substack{s_{i} \cdots s_{j}: \\
\sum_{s_{l}=s}}} 1 \leqq e^{s+j}, \\
\left(\begin{array}{c}
j+s \\
m
\end{array}\right) \leqq 2^{j+s}, \quad \sum_{m=j}^{j+s} e^{\alpha m} \leqq s e^{\alpha(j+s)} .
\end{gathered}
$$

Notice that the condition $\prod_{i \in K^{c}} \chi(|\operatorname{cl}(i)|>0)=1$ implies that $s \geqq j-k$.

Finally, for $\lambda r<e^{-(6+\alpha)}$ :

$$
\begin{aligned}
\left\|\bar{v}_{I}^{N}(t)\right\|_{1} & \leqq\left\|v^{N}\right\|_{\alpha} \frac{e^{\alpha j} e^{2 j}}{1-\lambda r e^{6+\alpha}} \sum_{k=0}^{j} \eta^{k}\left(\begin{array}{l}
j \\
k
\end{array}\right)\left(\lambda r e^{6+\alpha}\right)^{j-k} \\
& \leqq e^{\alpha j}\left\|v^{N}\right\|_{\alpha} \frac{e^{2 j}}{1-\lambda r e^{6+\alpha}}\left(\eta+\lambda r e^{6+\alpha}\right)^{j}
\end{aligned}
$$

We now fix all the parameters but $\alpha$. Choosing $t$ (and hence $r$ ) so large that $\eta=\frac{e^{-4}}{2}$ and, consequently $\lambda$ such that $\lambda r \leqq \frac{1}{2} e^{-2(6+\alpha)}$, we have

$$
\left\|\bar{v}^{N}(t)\right\|_{\alpha} \leqq 2 e^{-2}\left\|v^{N}\right\|_{\alpha} .
$$

We are now in position to comment on the estimates (4.11). In proving estimate (4.31) we have implicitly seen that the probability for a given particle to have an interaction cluster of large size is exponentially small, for $\lambda r$ small. Moreover each cluster is, with large probability, minimal, that is it is constructed by a minimal number of links. Finally the clusters are, with large probability, disjoint, since for an extra link we have to pay a price proportional to $N^{-1}$. This explains the meaning of estimates (4.11). For further details see (5.3) and (5.24) of [7].

Let us analyze now the term $R_{I}^{3}$ (see (4.17)). Notice that

$$
\begin{gathered}
\left\langle\left(\bar{f}^{N}(t) * \bar{g}^{N}(t)^{\perp}\right)_{I}, \varphi_{j}\right\rangle=\sum_{J \subseteq I}(-1)^{|J|} \sum_{S \subseteq I_{N} / J} \sum_{\substack{S_{1} \cdots S_{n}: \\
\bigcup_{i} S_{i}=S \\
S_{r} \cap S_{k}=\theta, r \neq k}} \sum_{R \subseteq I_{N} /(I / J)} \sum_{\substack{R_{1} \cdots R_{m}: \\
\bigcup_{i} R_{i}=R \\
R_{r} \cap R_{k}=\theta, r \neq k}} \\
\int d Z_{I \cup S \cup R} f^{N}\left(Z_{\frac{I \cup R}{j}}\right) g\left(Z_{J \cup S}\right) \mathbb{E}_{1} \mathbb{E}_{2}\left[\varphi_{j}\left(\left(T_{1}^{t} Z_{\frac{I \cup R}{J}}\right)_{I / J} ;\left(T_{2}^{t} Z_{J \cup S}\right)_{J}\right)\right. \\
\prod_{i \in I / J} \chi\left(\operatorname{cl}(i)=R_{i}\right) \chi\left(n\left(\bar{R}_{i}\right)=\left|R_{i}\right|\right) \\
\left.\prod_{i \in J} \chi\left(\operatorname{cl}(i)=S_{i}\right) \chi\left(n\left(\bar{S}_{i}\right)=\left|S_{i}\right|\right)\right]
\end{gathered}
$$

where $T_{i}^{t}, i=1,2$ are two identical independent copies of the same process $T^{t}$. $\mathbb{E}_{i}, i=1,2$ denote the expectations for the processes and $n=|J|, m=|I / J|$.

The difference with the expression (4.15) which defines $\bar{v}^{N}$ is twofold. In the expansion (4.32) $R \cup J$ and $S \cup(I / J)$ are not disjoint. Furthermore the product of the expectations $\mathbb{E}_{1} \mathbb{E}_{2}$ replaces the expectation $\mathbb{E}$. Following [7] we can control (see estimate (5.35) for the details) the contributions in (4.32) due to the terms with $R \cup J \cap S \cup(I / J) \neq \emptyset$. This contribution is bounded by $C^{j} / N$. An analogous 
estimate holds also for the difference between the product of the expectations and the expectation of the product. In conclusion:

$$
\left\|\left(\bar{f}^{N}(t) * \bar{g}^{N}(t)^{\perp}\right)_{I}-\bar{v}_{I}^{N}(t)\right\|_{1} \leqq \frac{C_{2}^{j}}{N} .
$$

Notice that the constants appearing in (4.11) and (4.33) are independent of $t$ and the parameters of the model. From (4.11), (4.33) and Proposition 3.2:

$$
\left\|v_{j}^{N}\right\|_{1} \leqq\left\|\bar{v}_{j}^{N}(t)\right\|_{1}+\frac{C_{3}^{j}}{N}
$$

for $\lambda$ small and a suitable constant $C_{3}>1$. Finally, choosing $\alpha=\log C_{3}$ we have

$$
\left\|v^{N}\right\|_{\alpha} \leqq 2 e^{-2}\left\|v^{N}\right\|_{\alpha}+\frac{1}{N}
$$

by (4.30). The proof is now complete since $2 e^{-2}<1$.

\section{Appendix A}

Proof of Proposition 3.1. Since

$$
K^{t} Z_{I}=\left\{K^{t} z_{i}\right\}_{i \in I}
$$

is a collection of independent one-particle stochastic processes, it is enough to prove that, for all $\eta>0$, there exists $t_{0}$ such that, for $t>t_{0}$, for all $u=u(z), u \in L_{1}$, satisfying $\int u d z=0$, then

$$
\left\|\bar{P}^{t} u\right\|_{1} \leqq \eta\|u\|_{1}
$$

Let $\bar{h}$ be the invariant probability measure for the Knudsen process, that is:

$$
\begin{array}{ll}
\bar{h}(x, v)=a H_{0}(v) & \text { for } v>0, \\
\bar{h}(x, v)=a H_{1}(v) & \text { for } v<0
\end{array}
$$

( $a$ being a normalization constant). Then (A.2) will be a consequence of the property:

$$
\lim _{t \rightarrow \infty}\left\|\bar{P}^{t} h-\bar{h}\right\|_{1}=0
$$

uniformly in all initial probability densities $h$. Indeed, denoting by $u^{+}$and $u^{-}$the positive and negative part of $u$ respectively, setting

we have

$$
\alpha=\int u^{+} d z=\int u^{-} d z,
$$

$$
\begin{aligned}
\left\|\bar{P}^{t} u\right\|_{1} & =\left\|\bar{P}^{t} u^{+}-\bar{P}^{t} u^{-}\right\|_{1} \\
& \leqq \alpha\left\|\bar{P}^{t}\left(\frac{u^{+}}{\alpha}\right)-\bar{h}\right\|_{1}+\alpha\left\|\bar{P}^{t}\left(\frac{u^{-}}{\alpha}\right)-\bar{h}\right\|_{1} \\
& \leqq 2 \alpha \eta \leqq \eta\|u\|_{1}
\end{aligned}
$$

for $t$ sufficiently large. 
To prove (A.4) we follow Arkeryd et al. [12] and check that, whenever there is a cutoff on the low velocities, the convergence (A.4) (proved in [12] for general Maxwellians) is indeed uniform in the initial datum $h$.

Notice that (A.4) cannot hold, in general, uniformly in $h$. Indeed if $h_{n}$ is a sequence of initial data approaching $\delta(x-1 / 2) \delta(v)$ as $n \rightarrow \infty$, we suddenly realize that the limit (A.4) is achieved with increasing difficulty as $n \rightarrow \infty$.

By formula (2.9) of [12]:

$$
\begin{aligned}
\left\|\bar{P}^{t} h-\bar{h}\right\|_{1} \leqq & \int_{0}^{1} d x \int_{x / t}^{(x+1) / t} d v h(x-v t, v)+\int_{0}^{1} d x \int_{0}^{(x+1) / t} d v H_{0}(v) \bar{J} \\
& +\int_{0}^{1} d x \int_{-x / t}^{0} d v H_{1}(v) \bar{J}+\int_{0}^{1} d x \int_{(x+1) / t}^{2} d v H_{0}(v)\left|\bar{J}+J_{-}\left(t-\tau_{x, v}\right)\right| \\
& +\int_{0}^{1} d x \int_{-2}^{x / t} d v H_{1}(v)\left|\bar{J}-J_{+}\left(t-\tau_{x, v}\right)\right|
\end{aligned}
$$

where

$$
\bar{J}=\frac{1}{\int_{1}^{2} H_{0}(v) d v+\int_{-2}^{-1} H_{1}(v) d v}
$$

is the flux of the stationary measure $\bar{h}, J_{-}(t)$ and $J_{+}(t)$ are the fluxes in 0 and 1 respectively and $\tau_{x, v}=-\frac{(1 / 2)(1-\operatorname{sgn} v)-x}{v}$ is the time of the free flight from the boundary to the point $x$.

Note that for $t>2$ the first three terms in the right-hand side of (A.7) are vanishing. To analyze the last two terms we use the following representation (see Appendix A of [12]) for the fluxes:

$$
\begin{aligned}
J_{ \pm}(t)= & \pm A_{ \pm}(t) \pm \int_{0}^{t} \beta(d \tau) A_{ \pm}(t-\tau) \\
& \pm \int_{0}^{t} \beta([0, \tau)) d \tau A_{ \pm}(t-\tau),
\end{aligned}
$$

where $\beta(d \tau)$ is a suitable measure on $[0, \infty)$, independent of $h$, and $A_{ \pm}(t)$ are given by:

where

$$
A_{ \pm}(t)=\phi_{ \pm}+v_{ \pm} * \phi_{\mp}(t)
$$

$$
\begin{aligned}
& \phi_{+}(t)=\int_{0}^{1 / t} v d v h(1-v t, v), \\
& \phi_{-}(t)=-\int_{-1 / t}^{0} v d v h(-v t, v), \\
& v_{ \pm}(t)=\frac{1}{t^{3}} H_{0,1}\left( \pm \frac{1}{t}\right)
\end{aligned}
$$


For $t>1, \phi_{ \pm}(t)=0$ and $A_{ \pm}(t) \neq 0$ only for $t<2$. Therefore, for $t>2$ :

$$
\begin{aligned}
J_{ \pm}(t)= & \pm \int_{t-2}^{0} t \beta(d \tau) A_{ \pm}(t-\tau) \\
& \pm \int_{t-2}^{t} \beta([0, \tau)) d \tau A_{ \pm}(t-\tau) .
\end{aligned}
$$

The last term is bounded from below and above by $\beta([0, t-2))$ and by $\beta([0, t))$ respectively (because of $\int_{0}^{2} d t A_{ \pm}(t) \leqq 1$ ). Since

$$
\beta([0, \infty))=\left[\int_{0}^{\infty} t N(t) d t\right]^{-1}=\bar{J},
$$

where $N=v_{+} * v_{-}$is the distribution density of the random time of flight from 0 to 0 (or from 1 to 1 ), we have

$$
\left|J_{ \pm}(t) \mp \bar{J}\right|=\int_{t-2}^{0} t \beta(d \tau) A_{ \pm}(t-\tau)+\omega(t),
$$

where $\omega(t)$ does not depend on $h$ and $\omega(t) \rightarrow 0$ for $t \rightarrow \infty$.

Therefore for $t$ large, after a suitable change of variable (see [12] (2.10)), the $4^{\text {th }}$ term in the right-hand side of (A.7) is bounded by

$$
\int_{0}^{t} d \tau\left|J_{-}(t-\tau)+\bar{J}\right| B_{+}(\tau)
$$

where

Finally

$$
B_{+}(\tau)=\int_{0}^{1 / \tau} d v H_{0}(v) .
$$

$$
\begin{aligned}
\int_{0}^{1} d \tau\left|J_{-}(t-\tau)+\bar{J}\right| B_{+}(\tau) & \leqq c \int_{0}^{1} d \tau \int_{t-\tau-2}^{t-\tau} d \sigma \beta(\sigma) A_{+}(t-\tau-\sigma)+c \int_{0}^{1} d \tau \omega(t-\tau) \\
& \leqq c(\beta[0, t-3))+c \int_{0}^{1} d \tau \omega(t-\tau)
\end{aligned}
$$

and the right-hand side of (A.19) goes to zero and is independent of $h$. The last term in (A.7) is of the same nature and can be handled analogously.

\section{Appendix B}

Proof of Proposition 3.2. To prove Proposition 3.2 we use the standard series expansion technique for the short time validity proof (see $[10,11])$. The main difference here is that, due to the diffusive boundary conditions, we do not have the conservation of the $L_{\infty}$ norm with respect to the free flow and this makes the proof a little more tricky.

We fix a time $\tau$ and $r$ the smallest integer for which $r \geqq 4 \tau+1$ (see (3.10)). In the sequel we shall assume that $t \leqq \tau$. 
We first derive an $L_{\infty}$ estimate for $g_{j}^{N}(t)$ which satisfies the following integrated version of the BBKGY hierarchy associated to Eq. (2.4):

$$
g_{j}^{N}\left(Z_{j}, t\right)=\left(P_{j}^{t} g_{j}\right)\left(Z_{j}\right)+\varepsilon(N-j) \int_{0}^{t} d t_{1} P_{j}^{\left(t-t_{1}\right)} C_{j, j+1} g_{j+1}^{N}\left(Z_{j}, t_{1}\right)
$$

where $P_{j}^{t}$ is the semigroup associated to the $j$-particle stochastic process $T^{t} Z_{j}$ (see (2.3)) and

$$
\begin{aligned}
C_{j, j+1} g_{j+1}^{N}\left(x_{1} v_{1} \cdots x_{j} v_{j}\right)= & \sum_{i=1}^{j} \int d v_{j+1}\left|v_{i}-v_{j+1}\right| \chi(i, j) \\
& \times\left\{g_{j+1}^{N}\left(x_{1} v_{1}, \ldots x_{i} v_{i}^{\prime}, \ldots x_{j} v_{j}, x_{i} v_{j+1}^{\prime}\right)\right. \\
& \left.-g_{j+1}^{N}\left(x_{1} v_{1}, \ldots x_{i} v_{i}, \ldots x_{j} v_{j}, x_{i} v_{j+1}\right)\right\} .
\end{aligned}
$$

Moreover

$$
g_{j}\left(z_{1} \cdots z_{j}\right)=\prod_{i=1}^{j} g\left(z_{i}\right)
$$

For the Markov semigroup $P_{j}^{t}$ we have the following representation (see (3.12)):

$$
P_{j}^{t} g_{j}=\mathbb{E}_{\Omega}\left[P_{j}^{t}(\omega) g_{j}\right]
$$

where

$$
\left\langle P_{j}^{t}(\omega) g_{j}, \varphi_{j}\right\rangle=\mathbb{E}_{B}\left(\int \varphi_{j}\left(T_{\omega}^{t} Z_{j}\right) g_{j}\left(Z_{j}\right) d Z_{j}\right) .
$$

On the other hand we have

$$
\begin{aligned}
P_{j}^{t}(\omega) g_{j}\left(Z_{j}\right)= & g_{j}\left(T_{\omega}^{-t} Z_{j}\right) \chi\left(t<\tau\left(Z_{j}\right)\right) \\
& +\left(P_{j}^{t-\tau\left(Z_{j}\right)}(\omega)\right) g_{j}\left(T_{\omega}^{-\tau\left(Z_{j}\right)} Z_{j}\right) \chi\left(t>\tau\left(Z_{j}\right)\right),
\end{aligned}
$$

where

$$
\tau\left(Z_{j}\right)=\sup \left\{s \geqq 0 \mid T_{\omega}^{-s} Z_{j} \in \Lambda_{j}^{0} \equiv(0,1)^{j} \times \mathscr{V}^{N}\right\}
$$

is the (deterministic, once $\omega$ is fixed) time of the first collision with the boundary in the past.

Notice that for $Z_{j} \in \Lambda_{j}^{0}$ the process $T_{\omega}^{s} Z_{j}$ is deterministic for a short positive or negative time (before the first collision with the boundary in the future or in the past respectively).

Since $T^{-\tau\left(Z_{j}\right)}\left(Z_{j}\right) \in \mathscr{F}_{j}^{(i)}$ for some $i \in I_{j}$, (B.6) becomes

$$
\begin{aligned}
P_{j}^{t}(\omega) g_{j}\left(Z_{j}\right)= & g_{j}\left(T_{\omega}^{-t} Z_{j}\right) \chi\left(t<\tau\left(Z_{j}\right)\right) \\
& +\chi\left(t>\tau\left(Z_{j}\right)\right) H_{a}\left(v_{i}\right) \int d v_{i}^{\prime} v_{i}^{\prime} P_{j}^{t-\tau\left(Z_{j}\right)}(\omega) g_{j}\left(\left(T_{\omega}^{-\tau\left(Z_{j}\right)} Z_{j}\right)^{\prime}\right)
\end{aligned}
$$

where $\left(T^{-\tau\left(Z_{j}\right)} Z_{j}\right)^{\prime}$ is the configuration on the boundary in which the outgoing velocity $v_{i}$ is replaced by the ingoing one $v_{i}^{\prime}$, and $a=0,1$, depending whether particle $i$ is in 0 or 1 respectively. The expression (B.8) can be iterated developing 
The Boltzmann-Grad Limit for 1-D Boltzmann Equation again $P_{j}^{-\tau\left(Z_{j}\right)}(\omega)$, so that we arrive to write:

$$
P_{j}^{t}(\omega) g_{j}\left(Z_{j}\right)=\sum_{k \geqq 0} P_{j}^{t}(\omega ; k) g_{j}\left(Z_{j}\right),
$$

where $P_{j}^{t}(\omega ; k)$ is the contribution to $P_{j}^{t}(\omega)$ due to the term with $k$ collisions with the boundary. It is easily seen by (B.8) that

$$
\left\|P_{j}^{t}(\omega ; k) g_{j}\right\|_{\infty} \leqq M^{k}\left\|g_{j}\right\|_{\infty}
$$

for some positive constant $M$.

Coming back to (B.1) we have

$$
\begin{aligned}
g_{j}^{N}\left(Z_{j}, t\right)= & \sum_{n \geqq 0} \varepsilon^{n}(N-j)(N-j+1) \cdots(N-j+n-1) \\
& \times \int_{0}^{t} d t_{1} \cdots \int_{0}^{t_{n-1}} d t_{n} P_{j}^{\left(t-t_{1}\right)} C_{j, j+1} P_{j+1}^{\left(t_{1}-t_{2}\right)} \cdots C_{j+n-1, j+n} P_{j+n}^{t_{n}} g_{j+n}\left(Z_{j}\right) \\
= & \mathbb{E}_{\Omega} \sum_{n \geqq 0} \varepsilon^{n}(N-j)(N-j+1) \ldots(N-j+n-1) \sum_{k_{1} \cdots k_{n}} \\
& \times \int_{0}^{t} d t_{1} \cdots \int_{0}^{t_{n-1}} d t_{n} P_{j}^{\left(t-t_{1}\right)}\left(k_{1}, \omega\right) C_{j, j+1} P_{j+1}^{\left(t_{1}-t_{2}\right)}\left(k_{2}, \omega\right) \cdots \\
& \times C_{j+n-1, j+n} P_{j+n}^{t_{n}}\left(k_{n}, \omega\right) g_{j+n}\left(Z_{j}\right) .
\end{aligned}
$$

Notice that the operator $C_{j, j+1}$ acts by joining a new particle, say $j+1$, in the same position of one of the group $I_{j}$, without changing any position of the particles of such a group. As an important consequence we realize that the right-hand side of (B.11) is vanishing whenever $\sum_{i=1}^{n} k_{i}$, that is the total number of collisions with the boundary, is too large. Actually $\sum_{k_{1} \cdots k_{n}}$ is subjected to the constraint

$$
\sum k_{i} \leqq(j+n)(2 t+1) .
$$

Moreover, we also have the obvious estimate

$$
\left\|C_{j, j+1} g_{j+1}\right\|_{\infty} \leqq 2 j\left\|g_{j+1}\right\|_{\infty} .
$$

Therefore, from (B.11), reminding that $r \geqq 4 t+1$ :

$$
\begin{aligned}
\left\|g_{j}^{N}(t)\right\|_{\infty} & \leqq \sum_{n \geqq 0} \lambda^{n} \sum_{\substack{k_{1} \cdots k_{n} \\
k_{i} \leqq(j+n)(2 t+1)}} \frac{(2 t)^{n}}{n !} j(j+1) \cdots(j+n-1) M^{(j+n) r}\left\|g_{j+n}\right\|_{\infty} \\
& \left.\leqq(2 M e)^{r}\|g\|_{\infty}\right)^{j} \sum_{n \geqq 0}\left((e M)^{r} r \lambda\right)^{n}\|g\|_{\infty}^{n}
\end{aligned}
$$

Thus for $(e M)^{r} r \lambda\|g\|_{\infty}<\frac{1}{2}$ :

$$
\left\|g_{j}^{N}(t)\right\|_{\infty} \leqq 2\left((2 e M)^{r}\|g\|_{\infty}\right)^{j}
$$

We now prove, by using the bound (B.15), the convergence of $g_{j}^{N}(t)$ to $g_{j}$ in the limit $N \rightarrow \infty$. Putting

$$
\Delta_{j}^{N}(t)=g_{j}^{N}(t)-g_{j}
$$


after some elementary algebra, we obtain

$$
\begin{aligned}
\Delta_{j}^{N}\left(Z_{j}, t\right)= & \varepsilon(N-j) \int_{0}^{t} d t_{1} \bar{P}_{j}^{\left(t-t_{1}\right)} C_{j, j+1} \Delta_{j+1}^{N}\left(Z_{j}, t_{1}\right) \\
& +\varepsilon \int_{0}^{t} d t_{1} \bar{P}_{j}^{\left(t-t_{1}\right)} G_{j} g_{j}^{N}\left(t_{1}\right),
\end{aligned}
$$

where $\bar{P}_{j}^{t}=\bar{P}_{I_{j}}^{t}$, is, as usual, the Knudsen semigroup and

$$
G_{j} g_{j}\left(Z_{j}\right)=\sum_{i<h}\left|v_{i}-v_{h}\right| \chi(i, h) \delta\left(x_{i}-x_{h}\right)\left(g_{j}\left(Z_{j}(i, h)^{\prime}\right)-g_{j}\left(Z_{j}\right)\right)
$$

(see (2.4) to recall the notation).

Iterating (B.17) we obtain

$$
\begin{aligned}
\Delta_{j}^{N}(t)= & \sum_{n \geqq 0} \varepsilon^{n}(N-j)(N-j+1) \cdots(N-j+n) \\
& \times \varepsilon \int_{0}^{t} d t_{1} \cdots \int_{0}^{t_{n-1}} d t_{n} \bar{P}_{j}^{\left(t-t_{1}\right)} C_{j, j+1} \bar{P}_{j+1}^{t_{1}-t_{2}} \cdots C_{j+n-1, j+n} \\
& \times \int_{0}^{t_{n}} \bar{P}_{j+n}^{t_{n}-t_{n-1}} G_{j+n} g_{j+n}^{N}\left(t_{n+1}\right) d t_{n+1} .
\end{aligned}
$$

Thus to estimate $\Delta_{j}^{N}(t)$ we are led to control:

$$
I_{j}(t) \equiv \int_{0}^{t} \bar{P}_{j}^{t-s} G_{j} g_{j}^{N}(s) .
$$

For $\bar{P}^{t}$ we have an analogous representation as (B.8) in which $T_{\omega}^{t}$ is replaced by the free stream $S^{t}$ (i.e. $\left.S^{t}\left(x_{1} v_{1} \cdots x_{j} v_{j}\right)=\left(x_{1}-v_{1} t, v_{1}, \ldots x_{j}-v_{j} t, v_{j}\right)\right)$ :

$$
\begin{aligned}
\bar{P}_{j}^{t} G_{j} g_{j}\left(Z_{j}\right)= & \left(G_{j} g_{j}\right)\left(S^{-t} Z_{j}\right) \chi\left(t<\tau\left(Z_{j}\right)\right) \\
& +\chi\left(t>\tau\left(Z_{j}\right)\right) H_{a}\left(v_{i}\right) \int d v_{i}^{\prime} v_{i}^{\prime} \bar{P}_{j}^{t-\tau\left(Z_{j}\right)}\left(G_{j} g_{j}\right)\left(\left(S^{\tau\left(Z_{j}\right)} Z_{j}\right)^{\prime}\right) .
\end{aligned}
$$

Using the obvious inequality

$$
\int_{0}^{t} \delta\left(x_{i}-v_{i} s-x_{h}+v_{h} s\right)\left|v_{i}-v_{h}\right| d s \leqq 1
$$

by (B.21) we obtain

$$
\left\|I_{j}(t)\right\|_{\infty} \leqq j^{2} M^{r j} \sup _{s \leqq t}\left\|g_{j}^{N}(s)\right\|_{\infty} .
$$

The term $j^{2}$ in (B.23) arises from the sum appearing in the definition of $G_{j}$ while the factor $M^{r j}$ is due to the collisions with the boundary, whose maximum number is bounded by $r j$.

Inserting estimate (B.15) in (B.23), we have

$$
\sup _{s \leqq t}\left\|I_{j+n}(s)\right\|_{\infty} \leqq 2(j+n)^{2}\left((2 M e)^{2 r}\|g\|_{\infty}\right)^{j+n} .
$$


By (B.19) we can estimate $\Delta_{j}^{N}$ as $g_{j}^{N}$ and, thanks to (B.24), we finally obtain

$$
\begin{aligned}
\left\|\Delta_{j}^{N}(t)\right\|_{\infty} & \leqq \varepsilon\left(2(M e)^{r}\right)^{j} \sum_{n \geqq 0}\left((M e)^{r} r \lambda\right)^{n} \sup _{s \leqq t}\left\|I_{j+n}(s)\right\|_{\infty} \\
& \leqq \varepsilon 2 j^{2}\left((2 M e)^{3 r}\|g\|_{\infty}\right)^{j} \sum_{n \geqq 0}\left((2 M e)^{3 r} r \lambda\|g\|_{\infty}\right)^{n} n^{2} \\
& \leqq \varepsilon 4 j^{2}\left((2 M e)^{3 r}\|g\|_{\infty}\right)^{j}
\end{aligned}
$$

for $r \lambda\|g\|_{\infty} M^{3 r}$ sufficiently small. Hence the proof is achieved.

Acknowledgements. It is a pleasure to thank J.L. Lebowitz for having suggested the problem and R. Esposito and E. Presutti for stimulating discussions.

\section{References}

1. Esposito, R., Lebowitz, J.L., Marra, R.: The Navier-Stokes limit of Stationary solutions of the nonlinear Boltzmann equation. J. Stat. Phys. (1994) (to appear)

2. Goldstein, S., Lebowitz, J.L., Presutti, E.: Mechanical Systems with stochastic boundaries. Colloquia Mathematicae Societatis, Janos Bolyai 27, Random Fields, Esztergorn, Hungary (1981)

3. Arkeryd, L., Cercignani, C., Illner, R.: Measure solutions of the steady Boltzmann equation in a slab. Commun. Math. Phys. 142, 285-296 (1991)

4. Arkeryd, L.: Some examples of NSA methods in kinetic theory. Lect Notes in Math 1551, Berlin, Heidelberg, New York: Springer, 1992

5. Arkeryd, L., Nouri, A.: A compactness result related to the stationary Boltzmann equation in a slab, with applications to the existence theory. (1994) (to appear)

6. Ianiro, N., Lebowitz, J.L.: Stationary nonequilibrium solutions of model Boltzmann equation. Found. of Phys. 15, 531-538 (1985)

7. Caprino, S., Pulvirenti, M.: A cluster expansion approach to a one-dimensional Boltzmann equation: A validity result. Commun. Math. Phys. 166, 603-631 (1995)

8. Caprino, S., De Masi, A., Presutti, E., Pulvirenti, M.: A derivation of the Broadwell equation. Commun. Math. Phys. 135, 443-465 (1991)

9. De Masi, A., Presutti, E.: Mathematical Methods for Hydrodynamical Limits. Lecture Notes in Math. 1501, Berlin, Heidelberg, New York: Springer 1991

10. Lanford, O.: Time evolution of large classical systems. Lecture Notes in Phys. Berlin, Heidelberg, New York: Springer 1975

11. Cercignani, C., Illner, R., Pulvirenti, M.: The mathematical theory of dilute gases. vol. 106, Berlin, Heidelberg, New York: Springer Appl. Math. Sci. 1994

12. Arkeryd, L., Ianiro, N., Triolo, L.: The trend to a stationary state for the Lebowitz stick model, Math. Meth. in the Appl. Sci. 16, 739-757 (1992)

Communicated by Ya.G. Sinai 
